\title{
Luminex Technology for Anti-HLA Antibody Screening: Evaluation of Performance and of Impact on Laboratory Routine
}

\author{
Maria Bernadette Colombo, ${ }^{1}$ Simone Elisabeth Haworth, ${ }^{1}$ Francesca Poli, ${ }^{1 *}$ Angela Nocco, ${ }^{1}$ \\ Giuseppe Puglisi, ${ }^{1}$ Annalisa Innocente, ${ }^{1}$ Marta Serafini, ${ }^{1}$ Piergiorgio Messa, ${ }^{2}$ \\ and Mario Scalamogna ${ }^{1}$ \\ ${ }^{1}$ Dipartimento di Medicina Rigenerativa, Fondazione Ospedale Maggiore, Policlinico, Mangiagalli, Regina \\ Elena, IRCCS, 20122 Milan, Italy \\ ${ }^{2}$ Dipartimento dell'Area della Medicina e Specialità Mediche, Fondazione Ospedale Maggiore, Policlinico, \\ Mangiagalli, Regina Elena, IRCCS, 20122 Milan, Italy
}

The recent introduction of new technologies such as Luminex has provided alternative methods to the Complement Dependent Cytotoxicity (CDC) test for HLA specific antibody detection. In this study we compared the results obtained with CDC to those obtained using a Luminex method with the aim of evaluating the impact of this new technology on antibody screening policies in our transplant setting.

A total of 1,421 sera, acquired from patients on the waiting list for a kidney transplant or following transplantation, were tested by both methodologies. CDC was performed using a whole lymphocyte population comprising a panel of 52 cells. The percentage panel reactive antibodies (PRA) and antibody specificity were evaluated using Lambda Scan Analysis software. For the Luminex method sera screening and identification of antibody specificity were carried out using the LABScreen Mixed and LABScreen PRA respectively.

The overall concordance between the results obtained using the CDC and the Luminex methods was $85 \%$. HLA antibody specificity was confirmed in $96 \%$ of the sera which tested positive using the Luminex system and serum positivity corresponded with a previous sensitisation event in these individuals. Using the Luminex method $18 \%$ of patients on the waiting list were considered and managed as sensitised as compared to $7 \%$ when testing with CDC alone. The Luminex method was able to detect a number of antibody specificities significantly more frequently than the CDC method and in addition the CDC method failed to detect some of the antibody specificities detected by the Luminex system.

Based on this comparison study we have incorporated the Luminex methodology into our screening strategy. $\odot 2007$ Clinical Cytometry Society

Key terms: complement dependent cytotoxicity (CDC) test; HLA specific antibody detection; Luminex technology

Since the first evidence in 1969 that a positive crossmatch in kidney transplantation is associated with hyper acute rejection of the transplanted organ (1), numerous studies demonstrating the clinical relevance of anti-HLA antibodies have been published (2-4). Because of the implementation of Complement Dependent Cytotoxicity test (CDC) cross-match testing in histocompatibility laboratories, hyper acute rejection has become a rare event.

For more than 40 years the CDC test was the only technique for detecting anti-HLA antibodies and it is still the most frequently used method for pre-transplant cross-match tests.

The introduction of less labor intensive techniques such as ELISA and Flow Cytometry prompted many laboratories to examine the new methodologies in comparison with $\operatorname{CDC}(5,6)$. One of the most consistent observations is that Flow Cytometry and ELISA are more sensitive with respect to standard serology, the former being able to detect complement fixing and complement

\footnotetext{
${ }^{*}$ Correspondence to: Francesca Poli, Dipartimento di Medicina Rigenerativa, Fondazione Ospedale Maggiore, Policlinico, Mangiagalli, Regina Elena, IRCCS-Via Francesco Sforza 35, 20122 Milan, Italy.

E-mail: france.poli@policlinico.mi.it

Received 14 September 2006; Revision 16 February 2007; Accepted 22 February 2007

Published online 30 March 2007 in Wiley InterScience (www. interscience.wiley.com).

DOI: 10.1002/cyto.b.20353
} 
nonfixing antibodies. The clinical significance of noncomplement fixing antibodies however is a subject of debate (7-11). Furthermore, the increased sensitivity of solid phase techniques has in turn increased the number of patients with a detectable sensitization previously considered as nonsensitized, which has resulted in repercussions in the management of waiting lists.

More recently a new methodology has become available in histocompatibility laboratories. This system uses micro beads coated with Class I or Class II HLA antigens and a flow analyzer. When appropriate a single antigen assay allows confirmation of antibody specificity.

We have introduced this technology, commonly known as Luminex, into our laboratory, and used it in parallel with the CDC test, for anti-HLA antibodies screening for patients awaiting kidney transplantation and kidney transplant recipients.

In this analysis we compared the results obtained with CDC to those obtained using Luminex with the ultimate aim of evaluating the impact of this new technology on antibody screening policies and on kidney allocation in our transplant setting (12). The objective was to identify an optimal antibody detection regimen which is both practical and sufficiently sensitive for our requirements.

\section{MATERIALS AND METHODS Study Design}

We analyzed a total of 1,421 sera from 1,395 patients belonging to two groups.

The first group consisted of 815 sera from 789 patients on the waiting list for a cadaveric kidney transplant. Of these 723 had never been transplanted and 66 had returned to the waiting list following graft failure.

The second group consisted of 606 patients who underwent kidney transplantation at least six months before the analysis (median 7 years; range 6 months29 years). The majority of these were primary transplants using kidneys from a cadaver donor; however 46 individuals had received a second, five individuals a third cadaver kidney transplant and 90 of the 606 transplants were performed using living donors.

Sera were collected from both groups primarily to enable a large number of sera to be analyzed. The analysis of the two groups was performed separately to maintain homogeneity of each group and in addition also permitted a comparison of the antibody profiles for each situation.

Sera were collected from July to December 2004 and all patients involved gave informed consent. Ethical approval for the study was granted on the condition that the sera collected were screened for the purpose of assessing patients for transplantation.

All kidney transplant recipients had a functioning graft at the time of the study and were under immunosuppressive therapy.

\section{CDC Methodology}

A total of 1,421 sera were screened by CDC using a whole lymphocyte population consisting of a panel of 52 cells from Caucasian blood donors incorporating the HLA-A,-B, and -DR antigens normally detected in our population. The CDC protocol used was the standard method as previously published (13). In essence recipients' sera $(1 \mu \mathrm{l})$ were dispensed onto Terasaki trays (One Lambda, CA, U.S.A.) and positive and negative controls included for the purpose of quality control. The negative control comprised sera from male untransfused, group $\mathrm{AB}$ blood donors. The positive control was pooled sera obtained from patients with a PRA greater than $80 \%$. The trays were either used immediately or stored at $-40^{\circ} \mathrm{C}$. Fresh donor cells $\left[1 \mu \mathrm{l}\right.$ of a $2 \times 10^{6} / \mathrm{ml}$ suspension in Hanks solution (Euroclone, Italy)] were added to the recipient' sera and the trays incubated at $22^{\circ} \mathrm{C}$ for 60 min. Rabbit complement $(5 \mu \mathrm{l})$ was added to each well and the trays incubated for a further $60 \mathrm{~min}$ at $22^{\circ} \mathrm{C}$. Finally a cell staining solution composed of acridine orange, ethidium bromide, and quenching ink (Fluoroquench, OneLambda, U.S.A.) was added to each well of the tray. The trays were read using a dual-phase fluorescent microscope (Leitz, Germany). Positive reactions were scored when the number of lysed cells in each well was $20 \%$ or more, negative reactions were scored for wells with $0-20 \%$ cell death. The number of lysed cells in the negative and positive control wells was monitored for each tray. The percentage of panel reactive antibodies (PRA) was calculated and the specificity evaluated with the support of LAMBDA SCAN ANALYSIS software.

\section{Testing for the Presence of IgM Isotype Antibodies}

Sera that gave a positive result with CDC but a negative result with the Luminex method were retested with and without dithiotritol (DTT) present to differentiate between IgM and IgG antibodies.

\section{Luminex Methodology}

Antibody screening and identification of antibody specificity were carried out using a LABScan 100 Flow analyser (Luminex Corporation, Texas, USA). The products utilized were LABScreen Mixed kit (One Lambda, CA, USA) and LABScreen PRA. The former method simultaneously detects Class I and Class II antibodies using micro beads coated with purified Class I and Class II HLA antigens and the latter establishes the percentage PRA and specificity for Class I and II. The tests were carried out according to the manufacturer's instructions and the analysis was performed with One Lambda software (HLA visual 1.1). The same lot number for the Luminex products was used for all sera to avoid any lot to lot variability.

The cut-off value was calculated using negative sera (Blood group $\mathrm{AB}$ sera from 27 un-transfused healthy males). A mean value and three standard deviations were calculated obtaining a cut-off value of 3.0 with an ambig- 
uous area from 2.5 to 3.0. The cut-off value was discussed with the manufacturers who advised that the cutoff value of 3.0 was appropriate. Sera with equivocal results (a value between 2.5 and 3.0) were considered borderline positive and therefore retested to elucidate any ambiguity.

\section{A Qualitative Comparison of the Sera Positive by the Luminex and CDC Methods}

The results for all sera that were positive for anti-HLAClass I antibody specificities by the Luminex method were compared to the results for the same sera tested by the CDC method to determine if any HLA Class I antibody specificities were consistently missed by the CDC method. Any specificities that were detected by the Luminex system but undetected in the same serum by the CDC method were recorded. The number of sera (i.e. incidences) where each particular specificity was detected by the Luminex and the CDC method was also noted in order to determine the frequency of detection of each antibody specificity for each method.

\section{A Comparison of Antigen Representation for Both the CDC and Luminex Methods}

To contrast antigen frequencies of the CDC panel (52 cells) to that of the Luminex LABScreen PRA system (55 microspheres) the representation of antigens most frequently observed in the Italian Caucasoid population was determined by expressing the number of cells/ microspheres expressing a particular antigen as a percentage of the total number of cells/microspheres used.

\section{Statistical Analysis}

To determine any statistically significant differences in the detection of HLA antibody specificities between the two methods the percentage of positive samples were compared using $2 \times 2$ contingency tables using $\chi^{2}$ and Fisher exact analysis with the level of significance set at $P<0.05$ and $95 \%$ confidence intervals (95\% CI). All analyses were performed using The SAS version 9.1 (Cary, NC, USA).

The study was performed in 4 steps:

1. All sera were tested for the presence of anti-HLA antibodies with CDC and with the LABScreen Mixed kit (One Lambda, 21001 Kittridge Street, Canoga Park, CA, USA) with the aim of comparing the results from the two screening methods.

2. All sera positive or borderline positive with LABScreen Mixed kit were re-tested with the LABScreen PRA to identify the antibody specificity and confirm the screening results.

3. The sera positive only with CDC were tested with and without DTT in order to exclude the presence of IgM isotype antibodies.

4. All samples with discrepant results for CDC test versus Luminex LABScreen PRA test were re-tested in order to eliminate any technical error.

\section{RESULTS}

In the waiting list patient group the total number of sera testing positive for the presence of anti-HLA antibodies with the Luminex protocol was 149/815 (18\%) versus $63 / 815(8 \%)(P<0.0001)$ with $C D C$ and similar percentages were found in the 606 transplanted patients $(19 \%$ vs. $8 \%)(P<0.0001)$. The CDC whole lymphocyte method did not identify any anti-HLA Class II antibodies specificities in any of the sera tested for both groups of patients whereas 191/1421 sera tested positive for antiHLA Class II antibodies with the Luminex method. Anti HLA Class I antibodies recognise HLA Class I antigens present on T lymphocytes and Anti HLA Class II antibodies recognise HLA Class II antigens present on B lymphocytes. The low numbers of B lymphocytes present in the whole lymphocyte population used in the CDC test are the likely explanation why anti-Class II HLA antibodies were not detected by this method.

All samples that tested positive with the CDC method but were negative with the Luminex LabScreen Mixed test were retested by the CDC method with and without DTT. In all cases $(n=32)$ the sera were negative following the DTT test. Because of the above observations only sera that tested positive for Class I by the Luminex method (antibodies of the IgG isotype only) were compared to sera that tested positive and were DTT resistant with the CDC method (likely to be IgG isotype only) in order to ensure a valid direct comparison. The analyses of the sera positive for Class I IgG anti-HLA antibodies from the group of patients on the waiting list and from the transplanted patients are shown in Tables 1 and 2 respectively. The anti-HLA Class II antibody data for the Luminex method and the results of the DTT testing for the CDC method are described below.

\section{Analysis of Sera Taken from Patients on the Waiting List}

Concordance between the results for the Luminex Screen and the CDC test was calculated as the number of samples that gave the same result (either negative or positive) by both methods expressed as a percentage of the total number of sera tested. The analysis of sera positivity shown in Table 1 identified a concordance of $92 \%$ between the Luminex and CDC methods for the identification of anti-HLA Class I IgG specific antibodies.

In terms of discordant results, 66 of the 788 sera (8.5\%) which tested positive by the Luminex screen test were negative when tested using the CDC test $(P<$ $0.0001)$. Conversely $7(0.9 \%)$ of the sera which tested positive by the CDC method were negative with the Luminex screen; however all 7 of these sera were negative following DTT treatment and therefore were reported in Table 1 as both CDC and Luminex negative for IgG isotype anti-HLA Class I antibodies.

The Luminex method detected anti-HLA Class II antibodies in $99(12 \%)$ of the 815 sera tested in this group of patients; $27 / 815$ (3\%) sera were positive for Class II only and $72 / 815$ (9\%) for both Class I and II simultaneously. Of the 149 sera testing positive the distribution 
Table 1

Comparison Between CDC and LABScreen Mixed Results for Anti-HLA Class I IgG Antibodies Detected in Sera from Patients on the Waiting List for a Kidney

\begin{tabular}{|c|c|c|c|}
\hline & CDC positive & CDC negative & Total \\
\hline Class I IgG only & N. (\%) & N. (\%) & N. (\%) \\
\hline $\begin{array}{l}\text { Luminex positive } \\
\text { Luminex negative } \\
\text { Total }\end{array}$ & $\begin{aligned} 56 & (7.1) \\
0 & (0) \\
56 & (7.1)\end{aligned}$ & $\begin{array}{l}66(8.5) \\
666(84.5) \\
759(92.9) \\
\end{array}$ & $\begin{array}{l}122(15.5) \\
666(84.5) \\
788^{b}\end{array}$ \\
\hline
\end{tabular}

${ }^{\mathrm{a}} P<0.0001$.

b27/815 sera (3\%) were positive for HLA Class II antibodies only and $72 / 815$ (9\%) for both Class I and Class II according to the Luminex methodology. The percentage values shown in Table 1 are expressed in relation to the total number of sera tested that were not Class II positive only i.e. 788.

for Class I only, Class II only, and Class I and II antibodies simultaneously was 34,18 , and $48 \%$ respectively.

\section{Analysis of Sera Taken from the Transplanted Patients}

The anti-HLA Class I antibody analysis demonstrated a concordance of $91 \%$ between the CDC and Luminex methods in the group of transplanted patients as shown in Table 2. Of the sera tested, 49/560 (8.7\%) were positive using the Luminex method but negative by the CDC test $(P<0.0001)$.

Of the 606 sera, 92 (15\%) tested positive for anti-HLA Class II antibodies with the Luminex method, 46/606 (7.5\%) for Class II only and 46/606 (7.5\%) for both Class I and II simultaneously. Of the total of 116 sera that tested positive the distribution for Class I only, Class II only and Class I and Class II antibodies simultaneously was 20,40 , and $40 \%$ respectively.

The number of DTT sensitive sera (i.e. IgM isotype antibodies were present) were higher in this group when compared to the group of patients on the waiting list; $25 / 606$ (4\%) $(P<0.0001)$ of sera tested positive with the CDC test and negative with Luminex but were negative with the CDC method following DTT treatment (vs. $7 / 788(0.9 \%)$ for the pretransplant group).

We also observed differences in antibody profile between the group of patients on the waiting list and the group of patients that had been transplanted. The two groups showed differences in the distribution of anti-HLA Class I and anti-HLA Class II antibodies. In particular in the transplanted patient group the percentage of sera positive for anti-HLA Class II antibodies only was higher when compared to the waiting list group (40\% vs. $18 \%)(P<0.0001)$.

\section{Analysis of Cut-off Value for Luminex Screen Test}

The total number of sera testing positive by the Luminex screen for all patients from both groups was 265, of these, 11 sera (4\%) were positive by the LABScreen Mixed test (i.e. had a value greater than the cut-off value of 3), but tested negative in a subsequent analysis with LABScreen PRA, therefore no anti-HLA antibody specificity was identified in these sera. Of the 68 sera that were borderline positive (i.e. had a value between 2.4 and 2.99) with the LABScreen Mixed test, 44 (65\%) were confirmed positive when tested with LABScreen PRA for anti-HLA antibody specificity. All 44 sera had a previous sensitization event (e.g., pregnancy, blood transfusion, or organ transplantation). Of the sera that had a reading above the cut-off value with the LABScreen Mixed Luminex technique $96 \%$ corresponded with the positive identification of HLA specific antibodies when tested with the Luminex LABScreen PRA.

\section{Comparison of the Detection of Anti-HLA Class I IgG} Isotype Antibodies by CDC vs. Luminex Methodologies

Although there is a high concordance rate for the detection of HLA Class I IgG isotype antibodies between the two techniques $(92 \%$ and $91 \%$ for the waiting list and transplanted patient groups respectively), the Luminex method detected anti-HLA Class I antibodies in an additional 115 sera which tested negative for antiHLA-Class I antibodies with the CDC technique. The differences in antibody detection between the CDC and Luminex method were statistically significant for both the waiting list and post-transplant patient groups $(P<$ 0.0001 Fishers exact test).

\section{A Qualitative Comparison of the Sera Positive by the Luminex and CDC Methods}

This analysis was performed for the transplanted group of patients as post-transplant follow-up data would be available to assess the clinical impact of the antibodies detected. A comparison of samples positive by both Luminex LABScreen Mixed and LABScreen PRA i.e. an antibody specificity was identified $(n=65)$ and where specificities were determined by the CDC panel $(n=$ 21) was carried out and the data are shown in Table 3.

Table 2

Comparison Between CDC and LABScreen Mixed Results for anti-HLA Class I IgG Antibodies Detected in Sera from Transplanted Patients ${ }^{a}$

\begin{tabular}{lccr}
\hline Class I IgG only & CDC positive N. (\%) & CDC negative N. (\%) & Total N. (\%) \\
\hline Luminex positive & $21(3.8)$ & $49(8.7)$ & $70(12.5)$ \\
Luminex negative & $0(0)$ & $490(87.5)$ & $490(87.5)$ \\
Total & $21(3.8)$ & $539(96.2)$ & $560^{\mathrm{b}}$ \\
\hline
\end{tabular}

${ }^{a} P<0.0001$

${ }^{\mathrm{b}} 46 / 606$ sera $(7.5 \%)$ were positive for HLA Class II antibodies and 46/606 (7.5\%) for Class I and II simultaneously according to the Luminex methodology. The percentage values shown in Table 1 are expressed in relation to the total number of sera tested that were not Class II positive only i.e. 560 . 
Table 3

Analysis of Antibody Specificities Detected in Sera by Luminex and $C D C$

\begin{tabular}{lcclccc}
\hline \multirow{2}{*}{$\begin{array}{l}\text { HLA-A } \\
\text { antibodies }\end{array}$} & \multicolumn{2}{c}{ Number of sera } & & HLA-B & \multicolumn{2}{c}{ Number of sera } \\
\cline { 2 - 3 } \cline { 5 - 6 } & Luminex & CDC & antibodies & Luminex & CDC \\
\hline A1 & 3 & 1 & B7 & 2 & 0 \\
A2 & 14 & 8 & B8 & 1 & 0 \\
A3 & 4 & 1 & B12 & 6 & 2 \\
A9 & 13 & 2 & B13 & 3 & 1 \\
A10 (25) & 6 & 2 & B14 & 1 & 0 \\
A11 & 8 & 2 & B21 (49) & 4 & 0 \\
A28 & 10 & 3 & B22 & 2 & 0 \\
A29 & 1 & 0 & B27 & 8 & 0 \\
A30 & 1 & 0 & B35 & 1 & 0 \\
A31 & 1 & 0 & B40 & 3 & 0 \\
A32 & 6 & 0 & B41 & 2 & 0 \\
A33 & 2 & 0 & B42 & 2 & 0 \\
& & & B47 & 2 & 0 \\
& & & B53 & 2 & 0 \\
& & & B67 & B81 & 2 & 0 \\
& & & & 2 & 0 \\
\hline
\end{tabular}

The above table compares the number of sera i.e. incidences when the Luminex system was able to detect a specificity to the CDC method.

${ }^{\text {a }}$ These specificities were not represented on the CDC panelsee Table 4. The antibody specificities highlighted in bold were detected statistically significantly more frequently with the Luminex method when compared to the CDC method. A number of antibody specificities were detected by Luminex but undetected by CDC despite the antigens being represented on the CDC panel.

For simplicity the comparison was limited to the antiHLA-A and anti-HLA-B antibodies detected and did not include anti-HLA-C antibodies as the consensus is that these antibodies are less clinically relevant. The data as recorded in Table 3 showed that some of the Class I antibody specificities were detected more frequently by the Luminex than the CDC method. In particular these were HLA-A2, -A9, -A28, -A32, HLA-B44, -B49, and -B27. When the detection rate for these specificities was compared for the two methods (the number of times the specificity was detected in the total number of sera tested, $n=$ 606), the Luminex method appeared to be statistically significantly more sensitive for the specificities HLA-A9 $(P=0.009)$, -A32 $(P=0.03)$, and HLA-B27 $(P=0.007)$. The frequencies of these antigens in the Italian Caucasoid population are 28,10 , and $4 \%$ respectively (www. allelefrequencies.net).

To address the question of whether these specificities were under-represented on the CDC cell panel Table 4 was consulted and the representation on the test panels (number of cells vs. number of beads was comparable for both methods).

\section{A Comparison of Antigen Representation for Both the CDC and Luminex Methods}

Table 4 showed that the representation of the antigens HLA-A9 (24\% vs. $21 \%$ ), and HLA-A32 (11\% vs. $13 \%)$ and the HLA-B27 (5\% vs. $2 \%$ ) panel antigen was comparable for both the Luminex and CDC techniques and not statistically significantly different. A number of antibody specificities were not detected in any sera by the CDC method despite the fact that the antigens were present on the test panel. Only three antibody specificities (HLAB53, -B67, and -B81) were identified by Luminex yet missed by CDC where the antigen was absent from the CDC panel and this occurred in two sera for each of these specificities.

\section{Analysis of Clinical Relevance of Antibodies Detected by the Luminex and CDC Methods}

In the series of transplanted patients, we found that $50 \%$ of subjects with positive sera had anti-HLA antibodies that were donor-specific. This value is comparable to the findings from a previous study (14). All subjects positive with Luminex technique alone $(n=188)$ had an anamnesis positive for immunological stimuli such as trans-

Table 4

The Percentage Representation of the Most Frequent Antigens Present in the Italian Population for Both the CDC Cell Panel and the Luminex Beads ${ }^{\mathrm{a}}$

\begin{tabular}{lrc}
\hline & \multicolumn{2}{c}{ Method used } \\
\cline { 2 - 3 } & CDC (\%) & Luminex (\%) \\
\hline HLA-A antigen specificity & 14 & 14 \\
1 & 52 & 27 \\
2 & $8^{\mathrm{b}}$ & $7^{\mathrm{c}}$ \\
28 & 19 & 11 \\
3 & 8 & 13 \\
11 & 23 & 24 \\
9 & 13 & 25 \\
$10^{\text {d }}$ & 42 & 47 \\
19 & & \\
HLA-B antigen specificity & 31 & 11 \\
5 & 21 & 5 \\
35 & 19 & 5 \\
18 & 17 & 5 \\
7 & 6 & 5 \\
8 & 19 & 9 \\
12 & 8 & 5 \\
13 & 6 & 9 \\
40 & 6 & 11 \\
14 & 13 & 16 \\
15 & 13 & 11 \\
16 & 9 & 11 \\
17 & 6 & 11 \\
21 & 4 & 16 \\
22 & 2 & 5 \\
27 & 6 & 4 \\
37 & 9 & 19 \\
\hline
\end{tabular}

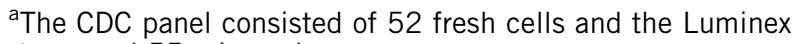
system used 55 microspheres.

${ }^{b}$ All 8 cells represented the A68 split of A28, A69 was not represented.

${ }^{\mathrm{C}}$ Both A68 and A69 splits of A28 were represented (An anti-A69 antibody was detected in only one serum by Luminex).

${ }^{\mathrm{d}}$ The CDC panel had only the A25 and A26 splits present wheras the Luminex panel had all A10 splits represented.

${ }^{\mathrm{e}}$ All A19 splits were represented by both the CDC and Luminex panels. 
fusions, failed transplants or pregnancies with the exception of five females and three males. Two patients who tested negative with the CDC test pre-operatively experienced acute rejection of their transplanted kidney. Retrospective analysis of the same sera using the Luminex system detected the presence of donor specific antiHLA Class I antibodies.

\section{DISCUSSION}

Individuals become sensitized following immunological stimuli such as previous blood transfusions, pregnancies, and failed transplants. Sensitization against HLA antigens becomes a concern when, because of end-stage organ failure, these individuals enter the waiting list for an organ transplantation. Sensitization against HLA antigens because of the presence of allo IgG antibodies not only prolongs waiting times but it has also been correlated with an increased risk of graft failure $(2,3)$.

Patient sensitization has traditionally been defined on the basis of PRA screening results generated from complement-dependent cytotoxicity assays and the level of sensitization is expressed by \%PRA. This method of definition has some limitations because it is dependent on panel composition and therefore several alternative or supplementary tests have been developed in the last decade. These new methodologies such as Flow Cytometry are based on solid-phase assays, and have demonstrated greater sensitivity and specificity with respect to CDC $(5,6,15)$. In our laboratory, clinical relevance, cost implications and staff resources have limited the use of Flow Cytometry to selected cases, namely pediatric subjects and living related transplants. Such limitations prompted us to assess an alternative technique hence our evaluation of the recently developed Histocompatibility and Immunogenetics application of Luminex technology. In terms of consumables, manpower and capital equipment we found Luminex technology less expensive and more user friendly than Flow Cytometry. In addition no particular expertise is needed for the data acquisition phase of the Luminex techniques and the discrimination between positive and negative reactions is simple and clear relative to the alternative screening methods currently available. Collection of results using both the Luminex and CDC methodologies for a large number of samples collected from patients on the waiting list and from patients who had received a kidney transplant provided considerable data for our retrospective study and allowed us to compare the two techniques to ascertain the optimum screening strategy.

The overall concordance between the two methods was $85 \%$ however this comparison was not appropriate as the two methods detect nonoverlapping antibody population i.e., our whole lymphocyte population CDC method detected IgM antibodies but did not identify anti HLA-Class II antibody specificity and the Luminex method detected both anti-HLA Class I and anti-HLA Class II antibody specificities but only of the IgG isotype. A direct comparison of the two methods for the detection of anti HLA Class I IgG antibodies alone revealed that the Luminex method identified HLA specificities in an additional $60 \%$ of the total number of sera which screened positive for anti-HLA Class I antibodies using the CDC method. According to the manufacturers, the Luminex LAbScreen Mixed test is more sensitive but less specific than the LABScreen PRA test, which resulted in 11 samples positive (cut-off above 3) by the screening test but negative for HLA-specificities i.e. false positives in our study. Similar 24/66 of the samples that screened borderline positive (cutoff-2.4-2.99) were negative by the LABScreen PRA test. This system prevents true positives being undetected and only resulted in a small percentage (4\%) of false positives. The analysis of the samples that had a borderline cut-off for the Luminex method, indicated that all samples with a borderline result plus a sensitization history can be routinely selected for further analysis with the LABScreen PRA test.

Qualitative analysis of the performance of the two methods demonstrated that the Luminex system was able to detect a number of antibody specificities significantly more frequently than the CDC technique although the representation of these antigens was comparable for the test panels of both methods. The three antibody specificities identified by the Luminex method but undetected by the CDC test where the antigens were absent from the CDC panel were all of rare frequency so unlikely to be included on a randomly selected CDC panel such as for this study but may be of clinical relevance. Therefore the Luminex panel not only allows better detection of antigens that are commonly represented in our patient population but prevents rare antibody specificities being missed.

These findings indicated that the screening and specificity detection approach of the Luminex system using the cut-off value as per this study is an accurate and sensitive method for the identification and determination of the nature of a positive reaction.

The higher percentage of sera positive for anti-HLA Class II antibodies profile observed in the transplanted patient group when compared to the group of patients on the waiting list ( $40 \%$ vs. $18 \%)$ as detected by the Luminex method may be a result of interaction between donor antigen presenting cells/Class II HLA molecules and recipient $T$ cells via the direct recognition pathway, typical of the early post-transplant phase. Monitoring of these patients post transplant requires an appropriately sensitive antibody protocol but in addition the clinical relevance of the antibodies needs to be clearly established. The analysis of the data of clinical relevance of the antibodies detected by the Luminex and CDC method indicated that the antibodies detected by Luminex alone were of clinical importance and corresponded with a sensitization event; however the CDC failed in two cases to detect antibodies crucial to transplant outcome. We are currently collecting data relating to graft outcome on all patients to determine whether the apparently increased sensitivity of the Luminex method observed in both groups of patients in this study impacts 
on graft and patient survival. Previous studies on this subject give conflicting results $(14,16)$.

The percentage of sera with IgM isotype antibodies present was also higher in the transplanted group when compared to the patients on the waiting list ( $4 \%$ vs. $0.9 \%$ ). This may be explained by the fact that the transplanted group of patients are immunosuppressed and as a result have more infections and in addition may have an increase in systemic inflammation following transplantation. Both of these factors may contribute to increased levels of IgM production, however the clinical relevance of the presence of IgM antibodies remains controversial $(5,17,18)$. Nonetheless the DTT integrated CDC method readily facilitates the detection of both the IgG and the IgM isotype antibodies within the same test.

This study provided not only quantitative but also qualitative analysis of the performance of our CDC method relative to the Luminex screening technique. Based on this comparison we have developed the following antibody screening strategy: all new patients entering the waiting list are tested with both CDC and Luminex LABScreen Mixed tests, and specificity definition analyzed as appropriate using Luminex LABScreen PRA. An autocross match and treatment of sera with DTT are also carried out on samples positive only with the CDC method. The CDC method is labor intensive and procurement of appropriate cells sometimes difficult however, we believe that the maintenance of $\mathrm{CDC}$ in addition to Luminex, allows greater confidence in cross-match results (as CDC is our method of choice for crossmatching prior to transplantation) and the ability to detect IgM in addition to IgG anti-HLA specific antibodies.

The advantages of the integrated use of Luminex and CDC are as follows:

1. Easy discrimination between antibodies specific for HLA Class I and Class II antigens.

2. Provision of information on acceptable mismatches in the cases of highly sensitized patients.

3. Increased rate of antibody detection when compared with CDC alone.

4. Avoids the need for B cell separation for CDC testing yet facilitates through detection of Class II specificities.

\section{ACKNOWLEDGMENTS}

We would like to acknowledge the Terasaki Foundation who kindly supplied a large proportion of the commercial reagents used in this study in recognition of our participation in the 14th International HLA and Immunogenetics Workshop.

\section{LITERATURE CITED}

1. Patel R, Terasaki PI. Significance of the positive cross-match test in kidney transplantation. N Engl J Med 1969;280:735-739.

2. Terasaki PI, Kreisler M, Mickey RM. Presensitization and kidney transplant failures. Postgrad Med J 1971;47:89-100.

3. Terasaki PI, Ozawa M. Predictive value of HLA antibodies and serum creatinine in chronic rejection: Results of a 2-year prospective trial. Transplantation 2005;80:1194-1197.

4. Terasaki PI, Cai J. Humoral theory of transplantation: Further evidence. Curr Opin Immunol 2005;17:541-545.

5. Worthington JE, Thomas AA, Dyer PA, Martin S. Detection of HLA specific antibodies by PRA-STAT and their association with transplant outcome. Transplantation 1998;65:121-125.

6. Pei R, Wang G, Tarsitani C, Rojo S, Chen T, Takemura S, Liu A, Lee J. Simultaneous HLA Class I and Class II antibody screening with flow cytometry. Hum Immunol 1998;59:313-322.

7. Slavcev A, Lacha J, Honsova E, Saidlova H, Lodererova A, Vitkos S, Valhova S, Striz I, Ivaskova E. Clinical relevance of antibodies to HLA antigens undetectable by the standard complement-dependent cytotoxicity test. Transpl Int 2003;16:872-878.

8. Gebel HM, Bray RA, Ruth JA, Zibari GB, McDonald JC, Kahan BD, Kerman RH. Flow PRA to detect clinically relevant HLA antibodies. Transplant Proc 2001;33:477.

9. Christiaans MH, Overhof R, ten Haaft A, Nieman F,van Hooff JP, van den Berg-Loonen EM. No advantage of flow cytometry cross-match over complement-dependent cytotoxicity in immunologically welldocumented renal allograft recipients. Transplantation 1996;62: 1341-1347.

10. Kimball P, Rhodes C, King A, Ham J, Posner M. Flow cross-matching identifies patients at risk for postoperative elaboration of cytotoxic antibodies. Transplantation 1998;65:444-446.

11. Scornik JC, Clapp W, Patton PR, Van der Wert WJ, Hemming AW, Reed AI, Howard RJ. Outcome of kidney transplants in patients known to be flow cytometry cross-match positive. Transplantation 2001;71:1098-1102.

12. Cardillo M, Poli F, Barraco F, De Fazio N, Rossini G, Boschiero L, Nocera A, Rigotti P, Marchini F, Zacchello G, Zanon G, Mandrini S, Chiaramonte S, Maresca C, Caldara R, Messa P, Berardinelli L, Ambrosini A, Montanaro D, Rampino T, Minetti E, Gotti E, Ghio L, Ginevri F, Albertario F, Scalamogna M. Renal transplantation. Strategies to prevent organ rejection-The role of an inter-regional reference center. Contrib Nephrol 2005;146:1-10.

13. Terasaki PI, McCelland JD. Microdroplet assay of human serum cytotoxins. Nature 1964;998-1000.

14. Vasilescu ER, Ho EK, Colovai AI. Alloantibodies and the outcome of cadaver kidney allografts. Hum Immunol 2006;67:597-604.

15. Pei R, Lee JH, Shih NJ, Chen M, Terasaki PI. Single human leukocyte antigen flow cytometry beads for accurate identification of human leukocyte antigen antibody specificities. Transplantation 2003;75: 43-49.

16. Morales-Buenrostro E, Buzo-Romero JM, de Leo C, Lopez M, OrtizArroyo VM, Perez-Garrido J, Herrera-Garcia C, Ganados J, Alberu J. Prevalence of HLA antibodies and its impact on graft function in a group of kidney transplant recipients: A cross-sectional study. Transplant Proc 2006;38:899-902.

17. Magee B, Martin J, Middleton D. The repercussions of implementing flow cytometry as a single HLA antibody screening technique in prospective renal transplant recipients. Transpl Int 2006;19:105109.

18. Tardif GN, McCalmon RT Jr. Successful renal transplantation in the presence of donor specific HLA IgM antibodies. Transplant Proc 1995;27:664-665. 